setting for a multi-disciplinary review of patients than does a traditional ward round when individual patients are interviewed by a whole team. Firstly it avoids the sort of intimidation that an individual patient might feel when confronted by several members of staff en masse-'much worse than vivas' according to your correspondent (Joumal, January $1978,132,112$ ). The group setting seems to enable patients to question staff in a way which is seldom seen in the traditional ward round. We feel that this approach is conducive to the sharing of decision- 1 making with patients and therefore therapeutic in itself; patients seem to be able to feel their way towards participating in making decisions without arousing too much staff anxiety, or insecurity in themselves. Secondly our type of approach is a way of enforcing rather than undermining the therapeutic momentum of self-help among patients-a force which I believe is potentially a highly effective tool in the treatment of psychiatric illness. Finally our ward round provides a suitable framework in which members of staff can examine their own motives and wishes during decision-making, thereby improving the quality of decisions.

Department of Psychiatry, Suman Fernando

Enfield District Hospital, Chase Wing, Enfield, Middx.

\section{CHRONIC ANXIETY IN ABORIGINALS}

Dear Sir,

Morice (1978), in an interesting approach to psychiatric illness among Australian Pintubi aboriginals, infers the existence of anxiety and depression from the presence of words in their vocabulary describing these affects. From his glossary it is apparent that these people include an inner feeling state, autonomic correlates, 'varying degrees of severity and differing provoking situations' as correlates. These observations are totally in accord with our experience of psychiatric symptoms in western desert aboriginals (Jones, 1972; Jones and Horne, 1973), but we called these phenomena 'fear'. We found very few examples of this state persisted for more than a few hours, although we drew our data from a survey of approximately 2,300 persons including some Pintubi. It will be noted that $\mathrm{Dr}$ Morice's glossary only includes descriptions of acute states. We concluded that chronic anxiety (which perhaps inadvisedly we called 'overt anxiety') was rare. His paper seems to give support to this proposition rather than refutation of it.

If these observations can be further supported they could be of value in highlighting a transcultural difference; presumably of developmental origin. Chronic states which might be anxiety equivalents do exist, for example hypochondriacal syndromes are common but these are usually quite free from autonomic correlates of anxiety.

Whether the proposition that chronic anxiety is infrequent in tribal aboriginals can now be established is, unfortunately, less than certain: even the most distant aboriginal groups are taking on white Australian ways and mores while losing their own culture. If the difference is real and culturally determined, one might expect more cases of anxiety to appear. If despite these social changes chronic overt anxiety remains infrequent, the question would be even more worthwhile investigating since it may indicate a biological difference. This difference in anxiety and the infrequent occurrence of homosexuality were the major findings in our studies. Most other psychiatric states were seen in recognizable form (Jones and Horne, 1972).

University of Melbourne, IVOR JONES

Department of Psychiatry, St Vincent's Hospital,

Fitzroy 3065,

Victoria, Australia

\section{References}

JONEs, I. H. \& HORNE, D. J. (1972) Diagnosis of psychiatric disorders among tribal Aboriginals. Medical foumal of Australia, 1, 345-9.

(1972) Psychiatric disorders of Aborigines of the Australian Western desert (ii). Social Science and Medicine, 6, 263-7.

— \& Horne, D. J. DE L. (1973) Psychiatric disorders among Aborigines of the Australian Western desert. Social Science and Medicine, 7, 219-28.

- \& Robinson, I. (1977) Severe illness with anxiety following a reputed magical act on an Australian Aboriginal. Medical Journal of Australia, 2, 93-6.

Morice, R. (1978) Psychiatric diagnosis in a transcultural setting. British Foumal of Psychiatry, 132, 87-95.

\section{BIOAGTIVE AMINES}

Dear Sir,

Research into central nervous system functions of different bioactive amines has yet to come up with some unifying hypotheses. I wish to propose an hypothesis that seeks to explain an apparent coincidence that substances released in skin injury (e.g. histamine) and vascular injury (serotonin) are also suspected of being regulator substances in the C.N.S.

I believe future research will demonstrate a general principle that, regardless of species or the particular amines released, those amine substances released from 